\title{
La Importancia de la Lectura en el Desarrollo de las Habilidades Investigativas del Estudiante Universitario
}

\section{The Importance of the Reading in the Development of the University Student's Investigative Abilities}

\author{
*Ana Verónica Osorio Sánchez ${ }^{1}$, Emma Yolanda Mendoza Vargas ${ }^{1}$, Erika Yessenia \\ Ballesteros Ballesteros ${ }^{1}$
}

${ }^{1}$ Universidad Técnica Estatal de Quevedo. Facultad de Ciencias Empresariales, Escuela de Marketing. Campus Ing. Manuel Haz Álvarez, km 1.5 vía Santo Domingo de Los Tsáchilas. Ecuador.

*aosorio@uteq.edu.ec

Fecha de recepción: 02/12/2017

Fecha de aceptación: 05/03/2018

Publicado: $29 / 06 / 2018$

\begin{abstract}
Resumen
La presente investigación persigue como objetivo fundamentar el hábito de la lectura como habilidad investiga en el proceso formativo de la educación superior, debido a que la formación del estudiante debe caracterizarse por el alto grado de independencia en la búsqueda de nuevos conocimientos. Una forma viable para cumplir el objetivo propuesto es mediante la lectura, con el propósito de mejorar las capacidades investigativas en las que sean capaces de formar posturas críticas y analíticas ante cualquier situación; así como proponer soluciones inmediatas a los problemas que más afectan al territorio de manera eficaz. Un reto de esta índole estimula al docente a prepararse mejor, a ser reflexivo y creativo, logrando alcanzar con el paso del tiempo independencia cognoscitiva. El estudio que se presenta hace uso de la metodología cualitativa sustentado del método etnográfico al ser una investigación descriptiva. Para el desarrollo del mismo se emplearon diversas técnicas como el análisis de documento, la entrevista y la observación participante, siendo la muestra intencional no probabilística y de fácil acceso. Los resultados alcanzados dan constancia de que el proceso docente educativo requiere de un reajuste en función de centrarlo hacia el
\end{abstract}


aprendizaje de los estudiantes, dejando a un lado las ataduras de la enseñanza tradicional e integrar los requerimientos de la nueva enseñanza en aras de incitar el deseo de autosuperación permanente en el aula para elevar el nivel científico estudiantil y así lograr que desarrollen habilidades investigativas mediante la profundidad y solidez de los conocimientos adquiridos.

Palabras clave: lectura, habilidades investigativas, tarea investigativa.

\begin{abstract}
The present investigation pursues as objective to base the habit of the reading like ability investigates in the formative process of the superior education, because the student's formation should be characterized by the high grade of independence in the search of new knowledge. A viable form to complete the proposed objective is by means of the reading, with the purpose of improving the investigative capacities in those that are able to form critical and analytic postures before any situation; as well as to propose immediate solutions to the problems that more they affect to the territory in an effective way. A challenge of this nature stimulates to the educational one to get ready better, to be reflexive and creative, being able to reach with the step of the time cognitive independence. The study that shows up makes use of the qualitative sustained methodology from the ethnographic method to the being a descriptive investigation. For the development of the same one they were used diverse technical as the document analysis, the interview and the participant observation, being not the intentional sample probabilistic and of easy access. The reached results give perseverance that the educational process requires of a readjustment in function of centering it toward the learning of the students, leaving to a side the ties of the traditional teaching and to integrate the requirements of the new teaching for the sake of inciting the desire of permanent self-improvement in the classroom to elevate the scientific student level and
\end{abstract}


this way to achieve that they develop investigative abilities by means of the depth and solidity of the acquired knowledge.

Keywords: reading, investigative abilities, investigative task.

\section{Introducción}

La educación superior en América Latina hoy, mantiene una búsqueda por encontrar vías, formas, sistemas de enseñanza que favorezcan el desarrollo de estrategias intelectuales, métodos, habilidades que permitan al individuo de manera independiente y con eficiencia, orientarse y resolver los disímiles y complejos problemas que exige el siglo XXI en el que estamos viviendo. Esta tendencia valora como fundamental la selección de contenidos de enseñanza, aquellos que favorezcan los vínculos con la práctica social global, por lo que pretende que los estudiantes apliquen sus conocimientos en los diferentes sectores de la sociedad. La misma acentúa la misión de las universidades como instituciones sociales que deben preservar, desarrollar y promover, a través de sus procesos sustantivos y en estrecho vínculo con la sociedad, la cultura de la humanidad.

Ante estas circunstancias, se hace necesario potenciar en las carreras, el hábito por la lectura como herramienta y habilidad investigativa del estudiantado, centrando los esfuerzos en orientar esta misión hacia procesos sustantivos como la docencia y la investigación dirigidos a preservar, promover y crear cultura. Sobre la base de las consideraciones la autora (Núñez, 2014, p.1) considera: “los jóvenes universitarios son quienes, teóricamente, más debiesen leer libros y revistas de impacto social; manifestando autonomía y capacidad crítica sobre las áreas en que se desenvuelve su formación académica para el desarrollo de sus actividades intelectuales”.

Desde esta posición, se afirma que la lectura es un medio de acceso a la información y uno de los caminos para contribuir a la generación de conocimiento. Actualmente se 
ha demostrado que si el individuo está capacitado y posee competencias lectoras, podrá adquirir con más facilidad competencias en búsquedas y por consiguiente tendrá mejores condiciones de desarrollarse en la sociedad actual. De este modo y si se fomenta el hábito de la lectura en estudiantes de cualquier especialidad, "llegarán a comprender su importancia una vez que accedan a nuevos conocimientos a través de los procesos de enseñanza - aprendizaje que le permitan reflexionar, realizar análisis, comparaciones, confrontaciones, entre otros elementos de validez que le faciliten mayores posibilidades de insertarse en el mundo laboral, de interactuar en sociedad y de desenvolverse como ciudadanos críticos y participativos” (González, 1998, p.73).

$\mathrm{Al}$ respecto se sostiene que en la medida que el estudiante desarrolle habilidades para manejarse con los recursos informativos (sin importar su soporte), se estará contribuyendo a la formación de lectores críticos y autónomos que estarán preparados para acceder a la información a través del buen uso de la lectura. De esta manera se incrementarán los conocimientos, la capacidad de concentración y comprensión, permitiendo compartir sus pensamientos. Por lo tanto, un brillante estudiante primero debe de ser un buen lector, ya que la mejor forma de aprender e investigar, es leyendo. De igual modo se distinguirá por poseer un amplio vocabulario y excelente ortografía; con facilidades para redactar perfectamente cualquier tipo de escrito dígase: ensayos, artículos, boletines, noticias, entre otros.

En este sentido, la investigación se hace pertinente toda vez que se manifiesta dicha relación como un proceso en sí que no se ve materializado hasta que logra el impacto en la sociedad de cada uno de los procesos universitarios, dando el grado de pertinencia de la universidad como institución social. La ejemplificación práctica de dicha relación demuestra los retos cada vez más crecientes de la preparación del docente a partir de la formación del futuro profesional. 


\section{Desarrollo}

\section{El Proceso de Enseñanza - Aprendizaje y las Habilidades Investigativas en el}

\section{Contexto Universitario}

El aprendizaje debe diseñarse como un proceso en espiral, que lleva implícito un aprendizaje grupal, o sea, la posibilidad de una reflexión conjunta entre profesor y estudiantes, que los lleve a ambos a construir y perfeccionar su conocimiento en respuesta a las exigencias del contexto social. Desde este punto de vista el proceso de enseñanza - aprendizaje debe contribuir al cambio social desde la promoción de la reflexión crítica y no asumir posiciones conformistas entre el estudiantado. Esto implica el llamado a la "pertinencia social" en la enseñanza, para que desde los contenidos programáticos se atienda a las problemáticas sociales, económicas y políticas de la realidad social, lo cual no puede estar separado del proceso docente educativo.

Ante estos dos procesos (enseñanza - aprendizaje y el docente educativo) las autoras consideran válido hacer un paréntesis ya que existe una conexión muy estrecha si tenemos en cuenta que en ambos la finalidad es modificar conductas a través de la enseñanza, manifestándose en este caso en la manera de desarrollar la lectura como una habilidad investigativa en los estudiantes, siendo una de las tantas formas de ir erradicando los elementos que caracterizan a la escuela tradicional. Sin embargo, se diferencian de acuerdo a que en el primero el principal protagonista es el alumno y el profesor cumple con una función de facilitador de los procesos de aprendizaje. Para el desarrollo de este proceso, el estudiante pone en marcha diversos mecanismos cognitivos que le permiten interiorizar la nueva información que se le está ofreciendo por parte del profesor y así convertirla en conocimientos útiles.

En cuanto al segundo: "es la integración holística y sistémica de la enseñanza y el aprendizaje de todos sus componentes junto con los niveles de asimilación, de 
profundidad $\mathrm{y}$ estructural, en sus tres dimensiones: educativa, instructiva $\mathrm{y}$ desarrolladora. En este sentido, parte de una institución docente y se proyecta en la sociedad, con el encargo de educar al colegial para la vida a partir de compromisos sociales, debiendo ser capaz de enfrentarse a nuevas situaciones y problemas que se les presenten y resolverlos en pos de transformar la sociedad. Su cumplimiento solo se hace posible mediante el método de enseñanza que establece la secuencia que el profesor desarrolla para lograr sus propósitos educativos, instructivos y desarrolladores" (Sosa, 2007, p.1-3).

Actualmente y en correspondencia con las pautas de los Sistemas Nacionales de Ciencia, Tecnología e Innovación, la Educación Superior en América Latina se está replanteando nuevas proyecciones enmarcadas en las políticas científicas. De esta manera la práctica de la investigación en el contexto universitario constituye un rublo importante en los procesos de formación en los estudiantes universitarios. Según las consideraciones de Alarcón (2015, p.11); Tünnermann (2008, p.410) la Educación Superior debe "fortalecer la identidad cultural y la capacidad de las universidades para construir y promover una nueva cultura académica que se sustente en una cultura de calidad". Dicha propuesta se adecua a las necesidades del contexto de los centros educativos atendiendo al carácter activo del sujeto en el proceso de adquisición del conocimiento y en la utilización de métodos en el proceso de aprendizaje.

En el ámbito universitario, la educación científica - tecnológica que se imparte, y en particular en las carreras de ingeniería, se encuentra muy ligada a enfoques eminentemente técnicos, donde predomina un tratamiento sesgado de las influencias recíprocas entre la ciencia, la tecnología y la sociedad. Aunque esta cuestión está presente en muchos de los debates que tienen lugar en la actualidad, en Latinoamérica la situación parece encontrarse en un punto de indefinición. Siguiendo la tendencia 
internacional, una primera salida a esta situación podría ser la inclusión, en los planes de estudio de las ingenierías, desde la perspectiva Ciencia - Tecnología - Sociedad (CTS) que permitiera la aproximación a las relaciones existentes hoy entre los componentes de ese acrónimo.

Sobre el proceso de gestión curricular desde las carreras de ingeniería "persiste la necesidad de sustentarse de una visión holística sobre el pensamiento más moderno y de los estudios realizados acerca del diseño curricular y del rol que en el mismo desempeña la interdisciplinariedad" (Castellanos, 2004). La educación holística concibe la formación de los ingenieros desde la percepción de una comunidad de aprendizaje que posibilite un método para aprender y enseñar. En esto es básica la integración de tres ejes principales: el objeto de la profesión y la formación de habilidades profesionales; el enfoque científico para la solución de problemas y la formación ética del ingeniero contemporáneo.

A partir de estos elementos, entre los rasgos esenciales que deben caracterizar al ingeniero como profesional se destacan según Arana (1999) y Cañedo (2004):

- Poseer un conocimiento profundo de las ciencias básicas, específicas y del ejercicio de la profesión, capaz e independiente y con una sólida formación teórica y científica general.

- Un profesional en estrecha vinculación con la industria que adquiera durante su preparación en la universidad, las habilidades profesionales básicas que le permitan resolver los problemas más generales y frecuentes de su entorno social.

- Un profesional más integral, versátil y flexible, cuya virtud fundamental sea su capacidad de autopreparación y adaptación a los cambios, para lo que debe: desarrollar su capacidad de comunicación, de manejo, procesamiento y 
utilización de la información científico - técnica, el dominio de la computación, el conocimiento de lenguas extranjeras, su formación económica, ecológica y humanista en general.

- Poseer una formación cultural capaz de desarrollar las relaciones humanas, para lo cual requiere de altos conocimientos profesionales, sociales, ambientales, información actualizada, valores y sentimientos, ética profesional y autoestima.

- Contar con un pensamiento lógico, heurístico, científico, sistémico.

Teniendo en cuenta las complejidades en que se presenta y se manifiesta hoy la realidad, la educación en ingeniería debe incorporar el enfoque interdisciplinario en el análisis y tratamiento de los problemas que se planteen. Por ser una profesión tan cercana a la solución de problemas de la práctica requiere de una formación cada vez más interdisciplinaria, integral, científica, práctica, social y humanista. Ello será posible, en la medida en que se perfeccione también su formación científica, tecnológica, ecológica, social, ética, entre otras.

Hoy se hace continua referencia, "en múltiples dominios académicos y científicos acerca de la necesidad no sólo de aprehender y asimilar conscientemente teorías, leyes, conceptos, etc., sino al mismo tiempo desarrollar "habilidades, competencias" que le permitan a los estudiantes asumir una actitud responsable en la solución científica de los problemas que surgen en diversas esferas de su práctica social” (Machado, Montes de Oca; Mena, 2008, p.161). Dado el cúmulo de conocimientos, las habilidades y competencias se dirigen a que los futuros profesionales asimilen modos de actuación necesarios y suficientes para que puedan utilizarlos en bien de la sociedad y en correspondencia con los más altos valores de la humanidad. 
Los sustentos metodológicos imprescindibles que enriquecen la propuesta que exponemos se encuentran en las reflexiones sobre el concepto "tarea" elaborado por Silvestre y Zilberstein, Álvarez de Zayas y la clasificación aportada según la función que persiguen dentro de dicho proceso (Machado, Montes de Oca; Mena, 2008, p.162). Dada su importancia es necesario realizar ciertas precisiones conceptuales relacionadas con la tarea y su relación con las habilidades debido a que en ese proceso urge una dinámica que mantenga la interacción permanente de los futuros profesionales con la categoría contenido y entre ellos mismos:

Según expresan Silvestre y Zilberstein (2000, p.49) “(...) la tarea docente puede ser portadora de las exigencias que, si las cumple, le permiten lograr un aprendizaje que no sea sólo reproductivo, sino que le garantiza un mayor éxito y estimula su interés".

Para A. de Zayas (1992), "la tarea es aquel proceso que se realiza en ciertas circunstancias pedagógicas con el fin de alcanzar un objetivo, es decir, es la acción que se desarrolla atendiendo a las condiciones y que encierra tanto lo inductor como lo ejecutor".

En términos más concretos resumimos, "si la acción, al ser sistematizada deviene en habilidad y en el proceso formativo la tarea se constituye en la acción misma, entonces la ejecución de tareas que tengan como objetivo la acción y que necesiten la realización de su sistema operacional traerá como resultado el desarrollo de habilidades" (Machado, Montes de Oca; Mena, 2009, p.162).

Partiendo de los criterios expuestos con anterioridad, se hace pertinente hacer una revisión hacia el interior del contenido de trabajo que cada profesor realiza ante las disciplinas y asignaturas que imparte para comprobar si se está cumpliendo con dicha habilidad como corresponde, de lo contrario debiesen diseñar tareas que se sustenten en la realidad y en las contradicciones permanentes del proceso laboral que lleven como 
objetivos cada una de las acciones correspondientes para que en el momento de realizarla, el estudiantado pueda ejecutarlas sin dificultad. En otras palabras, se trata de garantizar las condiciones necesarias para que se cumpla este propósito condicionado por el nivel de desarrollo cognoscitivo alcanzado por los estudiantes, los intereses de la sociedad y los suyos propios.

En este sentido, es válido resaltar el papel tan importante que posee la orientación de las tareas, pues es mediante ella donde "se concretan las acciones a realizar en la clase y fuera de ella, es decir los estudiantes aprehenden ejecutando las acciones que el docente concibe como concreción de su actividad en la clase, las cuales se presentan de esta forma. De modo que si se realizan tareas de manera frecuente y periódica, bajo determinadas condiciones, cada vez más complejas, con diferentes conocimientos pero cuya esencia es la misma, se logrará el dominio de la habilidad para solucionar problemas profesionales" (Machado, Montes de Oca; Mena, 2008, p.162-163).

Por estas razones, se considera necesario que las tareas que se presentan en la clase conformen un sistema que se caracterice por ser variadas, en tanto que existan actividades con diferentes niveles de complejidad según los niveles de asimilación del estudiante; la aplicación del conocimiento tanto a situaciones conocidas como no conocidas promoverán el esfuerzo y el quehacer intelectual del estudiante por la búsqueda constante.

Desde esta mirada, "las tareas que se le orienten a los estudiantes deben abarcar las habilidades investigativas integradoras, tales como Modelar, Ejecutar (Obtener, Procesar, Comunicar Información) y Controlar, entre otras de menos grado de complejidad”. (Machado, Montes de Oca; Mena, 2008, p.165). Dichas habilidades son un requisito para poder desarrollar otras de mayor grado de generalidad ya que no existe actividad científico-profesional al margen de su desarrollo, pues el futuro egresado 
necesita inevitablemente de ellas para poder actuar en cualquier circunstancia del entorno laboral.

De tal modo, en su esencialidad dichas tareas apuntan a las siguientes acciones como parte de su contenido según los siguientes autores:

-Modelar: observar la situación; precisar los fines de la acción; establecer dimensiones e indicadores esenciales para ejecutar la acción; anticipar acciones y resultados.

- Obtener: localizar; seleccionar; evaluar; organizar; recopilar la información.

•Procesar: analizar; organizar, identificar ideas claves; re-elaborar la información, comparar resultados.

- Comunicar: analizar la información; seleccionar la variante de estilo comunicativo según el caso; organizar la información; elaborar la comunicación.

-Controlar: observar resultados; comparar fines y resultados; establecer conclusiones esenciales; retroalimentar sobre el proceso y los resultados de la acción (Machado, Montes de Oca; Mena, 2008, p.165-166).

A los efectos de lo antes expuesto, las autoras coinciden con los criterios planteados por los autores antes citados al concebir a la clase universitaria de la siguiente manera:

"Actividad transformadora, enmarcada en el tiempo-espacio formativo, que propicia las relaciones e interacciones entre alumnos, alumnos-docentes y estos con la sociedad y sus procesos, a través de la tarea, para producir y reproducir la cultura en su sentido más amplio y para lograr, sobre esa base, el cumplimiento de los objetivos del proceso (colaborativo y personalizado) de aprendizaje" (Machado, Montes de Oca; Mena, 2008, p.173). 
De esta manera, se puede concluir que no sólo es importante que los alumnos aprendan teorías, leyes y conceptos sino la importancia de la educación basada en competencias, habilidades, hábitos y actitudes cimentados en la práctica, mediante la orientación de tareas integradoras en las que deban profundizar y desarrollar habilidades investigativas teniendo como punto de partida la lectura del tema específico a desarrollar.

\section{La Lectura: Una Vía para la Comunicación y el Desarrollo de las Habilidades Investigativas}

Las transformaciones que está viviendo el mundo asociado a los grandes avances de la ciencia, la tecnología y la información en estos tiempos, ha producido un nuevo contexto socio - histórico donde la humanidad cada día debe asumir los cambios y retos que le impone la sociedad de la información y el conocimiento. Dichos cambios han impactado al sistema educativo universitario estableciendo una nueva concepción filosófica en los docentes. Esta realidad conlleva "al surgimiento de nuevos requerimientos y prácticas de gestión en el estudiante para responder de forma eficiente y efectiva a los cambios que experimentamos hoy en día en este sector" (Hernández, 2010, p.1). Estas exigencias provocadas por la sociedad del conocimiento han tocado a las universidades y a todos sus miembros en el mundo, y particularmente en América Latina.

De esta manera, la sociedad de la información y el conocimiento nos está dejando muy claro, que "los ciudadanos del siglo XXI nos constituimos en los principales usuarios de estas transformaciones aceleradas a través de la generación, uso y difusión de la información, materia prima esencial del conocimiento humano. En estos escenarios, la lectura se constituye hoy mejor que nunca, en la llave de acceso a la 
sociedad del conocimiento, en virtud de que a través de ella conocemos, comprendemos, consolidamos, analizamos, sintetizamos, aplicamos, construimos y reconstruimos los saberes de la humanidad" (Gutiérrez, 2005, p.1).

Sin embargo, ¿ocurre así en todas las universidades?, veamos cómo se manifiesta de manera muy sintética a través de estos ejemplos:

En la educación superior mexicana, "la problemática lectora se hace presente en una gran mayoría de los estudiantes universitarios, según los resultados alcanzados en el estudio realizado por La Asociación Nacional de Universidades e Instituciones de Educación Superior (ANUIES) con el objeto de conocer quiénes son y qué hacen los estudiantes en su tránsito por la educación superior en el nivel licenciatura, tanto dentro como fuera del espacio universitario" (Gutiérrez, 2005, p.1).

Por su parte, la Universidad Estatal Amazónica (UEA) en Colombia, “estableció una revisión de los antecedentes sobre el estado actual y los efectos futuros de la lectura crítica como habilidad investigativa, obteniendo un escaso interés de los estudiantes por este tipo de lectura, sobre todo si se considera que en una parte de la misión de la (UEA) se reconoce que generar ciencia y tecnología, formar profesionales y científicos, implica atender las percepciones sociales y académicas” (Rivera; Modéjar, 2014, p.1).

En Ecuador, "la enseñanza de la lectura, el desarrollo de su hábito y de la potencialidad que hay en cada sujeto para ello es un problema pedagógico de gran envergadura, porque se relaciona con el desarrollo cultural del país. Esta situación es resultado de la estructura educativa que responde a un modelo educativo tradicional basado en el memorismo, poca o ninguna reflexión crítica de la realidad actual y la escasa práctica con textos de alto nivel académico limitados por los escasos recursos económicos para la adquisición de los mismos” (Rivera; Modéjar, 2014, p.1). 
En consecuencia con los ejemplos antes expuestos se aprecia un notable déficit de la lectura por parte de los estudiantes lo cual es alarmante. Para ello se debe hacer un llamado de alerta producto a la necesidad de concientizar a los jóvenes universitarios sobre la importancia de la temática en cuestión, demostrándole cómo repercute en la sociedad su bajo ahínco por la lectura como habilidad investigativa una vez que culminen los estudios y se inserten en una institución a trabajar.

Ante esta debilidad que tanto afecta a gran parte de las universidades, se deben crear alternativas para motivar al estudiantado a salir de ese estado tan pavoroso en el que se encuentra ya que "en el proceso de enseñanza-aprendizaje, la lectura constituye ser una actividad de gran importancia. Por lo tanto, los directivos y maestros son los máximos responsables de construir una escuela que de cabida a nuevas formas de colaboración y participación de todos los actores que integran la comunidad escolar; o sea, que brinde a sus estudiantes un aprendizaje significativo, que alimente su curiosidad natural, su gusto y hábito por el estudio, que enseñe de manera tal que ayude a todos sus estudiantes a encontrar vías provechosas y diversas de acceso al conocimiento, estableciendo para cada uno altas expectativas y alentándolos a realizar siempre el máximo esfuerzo" (Cervantes, 2009, p.4).

Ante estas circunstancias, diversas instituciones de la educación superior se han visto en la necesidad de articular respuestas acordes con las posibilidades que las circunstancias les imponen. En la Universidad de Quevedo, Ecuador, por ejemplo, está haciendo hincapié en la importancia de la lectura como habilidad investigativa. "Una de las preocupaciones que guía muchos de estos estudios es la búsqueda de explicaciones para entender los procesos de apropiación de la lectura y de su desarrollo en el camino de formar lectores; de esta forma se busca proponer metodologías que eleven los niveles de lectura en el estudiantado" (Alarcón; Fernández, s/f, p.50). 
Un caso particular son los estudiantes de primer año de la carrera de Ingeniería en Marketing al carecer de hábitos por la lectura y de una cultura de investigación, por lo que son muy bajas las investigaciones en esta esfera. Esta falta de tareas investigativas genera en el perfil profesional una ausencia del conocimiento de la realidad socioeconómica del país producto de que no existe una estrecha relación entre la docencia y la investigación, donde ambos se conviertan en el pilar del conocimiento científico, porque es en esta relación donde se generan los nuevos conocimientos y las transformaciones sociales, que permitirán el avance de la sociedad.

Sobre la base de las consideraciones anteriores se plantean las siguientes interrogantes: ¿Contribuye el claustro de la carrera de Ingeniería en Marketing a la formación del estudiante como lector? ¿Cuál pudiera ser la forma más viable para desarrollar habilidades investigativas en los estudiantes de la educación superior que cursan la carrera de Ingeniería en Marketing?

Para dar respuesta a estas preguntas se plantea que "todo proceso de enseñanzaaprendizaje se puede visualizar desde diversas perspectivas de acuerdo a las diferentes corrientes psicológicas, pedagógicas, sociológicas, etc., esto implica ver al docente y al alumno como seres sociales, integrantes de grupos, que buscan el abordaje y la transformación del conocimiento, por tal motivo es necesario proponer nuevas técnicas de enseñanza dentro del aula (...) Las técnicas deben ser basada en un método, claro está que ambos se deben tratar de adaptar y tomar forma con base en las metas a donde se quiere llegar. Para la aplicación de esta y el logro de los objetivos, el maestro debe de crear estrategias para estimular a los estudiantes por la lectura" (Cervantes, 2019, p.23).

Sin embargo no siempre ocurre así, pues en ocasiones "el alumno y el maestro no se percatan de la importancia de la lectura en la formación y de la función que ésta desempeña en la praxis educativa. Los maestros, sobre todo a nivel de educación 
superior, asumimos que los alumnos han desarrollado esta habilidad y por lo tanto no requieren de un apoyo adicional; no obstante, muchos estudiantes sí leen, pero se les dificulta ir más allá de lo textual como para realizar una interpretación y valoración de lo leído. Para nadie es un misterio el hecho que, en general, los jóvenes egresan de educación secundaria con un nivel de comprensión de lectura que no es el más adecuado para su nivel y tampoco tienen una relación estrecha con las bibliotecas escolares. Por lo que nos hemos dado cuenta de que nuestros estudiantes de educación superior, se han atiborrado de "conocimientos", pero no han aprendido y no han adquirido la habilidad de realizar lecturas a profundidad" (Alarcón; Fernández, s/f, p.51).

No obstante, a pesar de presentar estas deficiencias, el claustro de profesores está perfeccionando los modelos académicos para desarrollar competencias de lectura crítica donde los estudiantes sean autónomos y protagonistas de su proceso de aprendizaje a su vez de tener la capacidad para autoevaluar su desempeño. Se han replanteado medidas a tomar en calidad de impartición de la docencia, la cual se ha estado efectuando mediante el control, las visitas y evaluaciones a clases por los directivos y jefes de departamentos.

Otro elemento que se está teniendo en cuenta para contrarrestar esta debilidad son los programas curriculares académicos, los cuales se están perfeccionando ya que la docencia "debe estar apegada a las exigencias reales de la sociedad, de integrar en sus planeaciones los elementos que le permitan actualizar el conocimiento de acuerdo al contexto en el que se encuentren para dar más atractivo a sus clases, que generen más interés, y sobre todo que intente hacer de la educación convencional una educación sistémica, recordemos que todo proceso educativo busca que el alumno desarrolle su personalidad libremente, por tal motivo se empeña en la transmisión de conocimientos, 
valores, actitudes y aptitudes que contribuyan a la transformación de cada individuo a ser cada vez mejor" (Cervantes, 2009, p.2).

Además se está potenciando con mayor ímpetu las tareas investigativas en las clases para que el estudiantado se vea en la necesidad de recurrir a una consulta bibliográfica que le permita aplicar los procedimientos de aprendizaje teórico en investigaciones que den soluciones en la práctica a los principales problemas sociales que afectan a sus territorios, para ello deben de desarrollar la habilidad lectora crítica del proceso educativo.

Teniendo en cuenta las dificultades presentadas, lo que se está realizando y lo que aún queda por hacer, no cabe duda que la lectura es una de las metas fundamentales de la enseñanza superior, considerada como una de las "habilidades prioritarias que hay que dominar al ser la base del aprendizaje y la puerta para la cultura". (Burón, 2000, p.13). "La falta de esta habilidad suele ser una gran limitante en el proceso de aprendizaje; esto es, cuando sólo se es capaz de leer en sentido técnico del término, pero se muestran dificultades para aplicar la lectura como herramienta para profundizar y ampliar los conocimientos" (Alarcón; Fernández, s/f, p.48).

En cuanto a las capacidades investigativas de los estudiantes de la carrera a la cual se hace referencia se deben de seguir perfeccionando los aspectos básicos de la investigación interpretativa y socio crítica, la elaboración de diagnósticos sociales y el diseño de proyectos de investigación para que puedan poner en práctica los conocimientos adquiridos. Al mismo tiempo que se deben de seguir diseñando, estructurando e insertando un conjunto de estrategias didácticas que estimulen la investigación en el estudiantado.

A partir de las soluciones a las interrogantes realizadas con anterioridad, podemos concluir diciendo: nos queda de tarea seguir promocionando la investigación en el 
trabajo social, para que se formen profesionales con capacidad y compromiso, con la generación de conocimientos sobre su quehacer profesional en una realidad social determinada. Para ello es necesario trascender los planes de estudio y hacer de la investigación una actividad permanente, que genere una cultura de investigación en la formación profesional en las universidades.

A propósito de lo abordado se sugiere una revisión hacia el interior de la universidad en cuanto al claustro que lo conforma, pues no siempre se cuenta con trabajadores profesionales que tengan dominio de los procesos docentes e investigativos; de lo contrario, respondieran a la realidad de este tiempo en cuanto a los avances de la ciencia, erradicando la postura presentada.

\section{Conclusiones}

El fomento del hábito de la lectura como herramienta y habilidad investigativa en el proceso formativo de la educación superior permite desarrollar la habilidad para la búsqueda en bases de datos y el trabajo con los gestores bibliográficos.

El hábito por la lectura como habilidad investigativa en el contexto universitario, permite la independencia cognoscitiva y la creatividad de los estudiantes; contribuye a la formación de lectores críticos, a perfeccionar los conocimientos en respuesta de las exigencias del contexto social mediante la investigación, a desarrollar competencias, al logro de la autonomía; potencia el vocabulario, la redacción, la ortografía y la cultura general e integral.

- Los planes de estudio de cada carrera de Ingeniería en Marketing deben de estar aparejados a las nuevas exigencias del siglo XXI, reorientados a través de las actividades curriculares y extracurriculares hacia el fortalecimiento del hábito de la lectura, contribuyendo a que la formación del estudiante universitario sea más competente. 
- Los estudiantes de la carrera de Ingeniería en Marketing no sienten interés por desarrollar investigaciones de la realidad de los contextos regionales, locales y nacionales del país y manifiestan entre las causas, según las entrevistas realizadas, la carencia la calidad con que reciben esa formación en la carrera. Esto hace ver que el profesional no sea destacado en el ámbito investigativo.

\section{Referencias Bibliográficas}

Alarcón Ortíz, R. (2015). “La educación superior en el Sistema Nacional de Ciencia, Tecnología e Innovación”. Conferencia realizada con motivo del encuentro con la Red Global para el aprendizaje, la innovación y la formación de capacidades. (GLOBELICS), Octubre, Cuba.

Alarcón Pérez, Lilia Mercedes y Fernández Pérez, Jorge A. (s/f). "La lectura crítica como herramienta básica en la educación superior”. En: Revista de la Facultad de Filosofía y Letra.p.4-7.

Álvarez de Zayas, C. M. (1992). La escuela en la vida. Editorial Félix Varela, La Habana, Cuba.

Arana, M. (1999). La cultura tecnológica en el ingeniero y el cambio de paradigma. En Tecnología y Sociedad. Editorial Félix Varela. La Habana, Cuba.

Castellanos, J. (2004). "Diseño conceptual para la implementación de un currículo holístico en la formación del ingeniero mecánico”. II Taller Internacional de Docencia Universitaria. Universidad de Cienfuegos. Cuba.

Cañedo, C. (2004). "Estrategia didáctica para contribuir a la formación de la habilidad realizar el paso del sistema real al esquema de análisis en el ingeniero mecánico”. [Tesis defendida en opción al grado científico de Doctor en Ciencias Pedagógicas]. Universidad de Cienfuegos. Cuba. 
González Morales, Alfredo. (1998). "Diagnóstico y efectividad motivacional en la promoción de la lectura”. En: Revista Isla, N.16, p. 73-76. Editorial Feijóo. Santa Clara, Cuba.

Gutiérrez Valencia, Ariel. (2005): "La lectura: una capacidad imprescindible de los ciudadanos del siglo XXI. El caso de México". En Revista Anales de Documentación, No. 8, julio 2005, p.91-90.

Hernández, Ysmael. La importancia de la formación investigativa en los docentes universitarios en el siglo XXI. [En línea].2010 Disponible desde internet en: http://www.gestiopolis.com/importancia-formacion-curricular-docentesuniversitarios-siglo-21/. Consulta el 19/12/2016

Machado Ramírez, Evelio F.; Montes de Oca Recio, Nancy y Mena Campos, Alodio. (2008). "El desarrollo de habilidades investigativas como objetivo educativo en las condiciones de la universalización de la educación superior”. En: Revista Pedagogía Universitaria, No. 1, enero 2010, p.22-28.

Machado Ramírez, Evelio F y Montes de Oca Recío, Nancy. (2009). "El desarrollo de habilidades investigativas en la educación superior: ABSTI”. En: Revista Humanidades Médicas, No.3, marzo-mayo 2009, pp.18-26.

Núñez Sobrino, Dulce María. (2014). "Estrategia Sociocultural para la Promoción de la Lectura en estudiantes de la comunidad universitaria Carlos Rafael Rodríguez”. Tesis defendida en opción al título de Máster en Estudios Socioculturales. Universidad de Cienfuegos, Cuba.

Rinvera García, Christian Geovany y Mondéjar Rodríguez, Juan. (2014): "La lectura crítica como habilidad investigativa en estudiantes universitarios". En: Revista Amauta. No.23, p.163-176. 
Silvestre O; M. \& Zilberstein T; J. (2000). ¿Cómo hacer más eficiente el aprendizaje. Instituto Central de Ciencias Pedagógicas? [formato electrónico].

Tünnermann Bernheim, C. (2008): “La educación superior en América Latina y el Caribe: diez años después de la Conferencia Mundial de 1998”, Editorial Javeria. Colombia.

Cervantes Villegas, Jesús A. ¿Cómo fomentar el hábito de la lectura y la comprensión lectora en los niños? [En línea]. 2009. Disponible desde internet en:http://vinculando.org/educacion/como_fomentar_habito_de_lectura_y_compre nsion_lectora.html. Consultado el 28/5/2017.

Sosa Flores, Miguel. El proceso docente educativo: Sus componentes. [En línea]. 2007. Disponible desde internet en: https://www.ecured.cu/Proceso_Docente_Educativo. Consultado el 28/5/2017. 